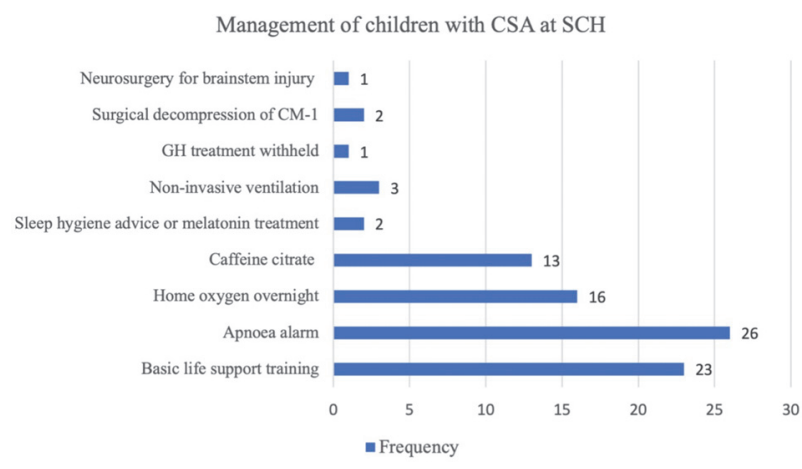

Abstract 9 Figure 1 Bar chart showing the frequency of different managements seen in children with CSA at SCH

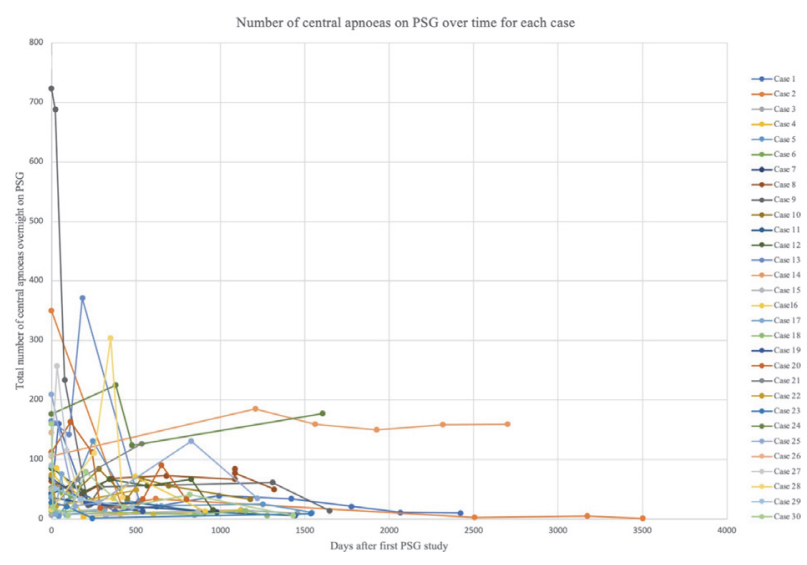

Abstract 9 Figure 2 Number of central apnoeas recorded on PSG over time for each child with CSA seen at $\mathrm{SCH}$, demonstrating a mean decrease of 15.42 central apnoeas from first to last PSG $(p=0.000)$

presentation, clinical events, diagnosis, investigations (including PSG and oximetry results), management (figure 1), factors related to management choices, and outcomes.

Results $43.3 \%$ presented with a 'brief resolved unexplained event' (11 resuscitated). The most common age at presentation was 8 -weeks $(n=8) .20 \%$ were being safeguarded. The most common co-morbidities $(n=9)$ were reflux and heart murmurs. The number of central apnoeas, AHI, and $3 \%$ desaturation index were shown to significantly improve from the first to last PSG ( $\overline{\mathrm{x}}$ improvement $=15.42, \mathrm{p}=0.000 ; \overline{\mathrm{x}}$ improvement $=15.80, \quad \mathrm{p}=0.002 ; \quad \overline{\mathrm{x}} \quad$ improvement $=15.82$, $\mathrm{p}=0.005$ respectively) (figure 2 ). $8 / 30$ patients were discharged from the sleep service ( $1 / 8$ deceased due to CSA). Children with parents reported to be anxious remained in the service for significantly longer than children without $(\mathrm{x} 2=3.855, \mathrm{p}=0.05)$.
Discussion PSG results demonstrated that this is a significant syndrome with risk of mortality, however the natural clinical course of paediatric CSA is improvement with time. Parental anxiety significantly increases the length of management time. This is the first study to document the death of a child primarily due to CSA.

\section{CONTINUOUS POSITIVE AIRWAYS PRESSURE (CPAP) - DRIVE THRU COLLECTION CLINIC (DTCC)}

Karena Cranstone*, Mark Unstead, Lauren Kimberley, Emily Seaman, Jessica Swan, Manisha Perera, Bethany Swaffield, Natalie Wilson, Harry Kirby, Stephanie Ferris. Royal Berkshire NHS Foundation Trust, Reading, UK

\subsection{6/bmjresp-2021-bssconf.8}

Introduction With a $2 \%$ increase in sleep referrals and impact from COVID-19, a safer and more effective way of working was required. Historically CPAP is issued face-to-face $(\mathrm{F} 2 \mathrm{~F})$, DTCC was introduced to increase patient capacity. The aim is to compare compliance and efficacy between DTCC and F2F appointments.

Method Between 12/11/20 \& 18/3/21 N=58 (44仓̊ \& 14ㅇ) patients with confirmed Obstructive Sleep Apnoea (OSA) attended DTCC. Average Oxygen Desaturation Index (ODI) 15.45 (4.47-46), age 52.1yrs (29-86), BMI 31.92 (21-55), Mean SpO2 93.81\% (81.68-96.69). Prior to DTCC patients watched demonstration video, paperwork was completed and machines pre-assigned. 10 minute appointment, patients were consented to AirView, mask size measured and post CPAP questionnaire plus brief instructions were given. First review at $\sim 4$ weeks, (use of $\geq 4 \mathrm{hrs} / \mathrm{p} / \mathrm{n}^{1}$ Weaver, TE) indicated compliance. Post ESS obtained and absolute $\Delta$ in ESS calculated to identify clinical outcomes. Data was analysis using Microsoft Excel.

Results Compliance of 50\% was achieved, $\mathrm{N}=29$ (22 $\widehat{\circ} 7$ \%), 29 patients either returned the machine, did not use or were non-compliant; (22 $\hat{\jmath} \& 7$ \% ). ESS reduced by 5.36 (50.93\%), however only 35 post CPAP questionnaires/ESS were returned. Average time for F2F appointment ( $\sim 5$ mins.) compared to DTCC ( 15 mins.). (Table 1$)$.

Discussion DTCC shows small reduction in compliance when compared to $61 \%$ from data previous local study. ${ }^{2}$ Therefore DTCC is a time efficient alternative to F2F appointments with average reduction of $\sim 30$ minutes per patient. The DTCC will aid recovery post COVID, allowing increased outpatient capacity while reducing footfall.

\section{REFERENCES}

1. Weaver TE, Grunstein RR. Adherence to continuous positive airway pressure therapy, proceedings of the american thoracic society 2008;5:173-178.

2. EJ Oakham, M Unstead, AD McGown. CPAP compliance and symptomatic benefit: a comparison of patients established on CPAP from GP or preoperative screening. Thorax 2017;2018:A1-A220

Abstract 10 Table 1 Results and key outcomes

\begin{tabular}{|c|c|c|c|c|c|c|c|}
\hline $\begin{array}{c}\text { Avg. hrs used } \\
\text { per night }\end{array}$ & AHI & Pre ESS & Compliant & $\begin{array}{c}\text { Post } \\
\text { ESS }\end{array}$ & $\begin{array}{c}\text { Absolute } \\
\Delta \text { ESS }\end{array}$ & $\begin{array}{c}\% \Delta \text { in } \\
\text { ESS }\end{array}$ & $\begin{array}{c}\text { Average time per } \\
\text { patient (mins.) }\end{array}$ \\
\hline 3.91 & 6.16 & 10.58 & $\mathrm{n} 29=50 \%$ & 5.36 & -5.58 & 48.97 & -30 \\
\hline $0-15.3$ & $0-66.3$ & 1 to 22 & $44 \AA \& 7 \%$ & 0 to 16 & $0-16$ & $0-100$ & DTCC $=15$ \\
\hline 2.99 & 11.05 & 4.96 & & 4.00 & 4.05 & 28.44 & $\mathrm{~F} 2 \mathrm{~F}=45$ \\
\hline
\end{tabular}

